\title{
UNMANNED AERIAL VEHICLE (UAV) DERIVED NORMALISED DIFFERENCE VEGETATION INDEX (NDVI) AND CROWN PROJECTION AREA (CPA) TO DETECT HEALTH CONDITIONS OF YOUNG OIL PALM TREES FOR PRECISION AGRICULTURE
}

\author{
Stanley Anak Suab ${ }^{1}$, Mohd Shahrizan Bin Syukur ${ }^{2}, \operatorname{Ram}_{\text {Avtar }^{1}} \&$ Alexius Korom ${ }^{2}$ \\ ${ }^{1}$ Faculty of Environmental Earth Science, Hokkaido University, Sapporo, Japan. stan@ eis.hokudai.ac.jp ; \\ ram@ees.hokudai.ac.jp \\ ${ }^{2}$ Faculty of Plantation and Agrotechnology, Universiti Teknologi MARA (UiTM) Sabah Branch, Kota Kinabalu, Sabah, \\ Malaysia.shahrizansyukur@gmail.com; alexi502@uitm.edu.my
}

KEY WORDS: Unmanned Aerial Vehicle (UAV), Normalised Difference Vegetation Index (NDVI), Crown Projection Area (CPA), Health conditions, Young Oil Palm

\begin{abstract}
:
Malaysia currently is one of the biggest global producers and exporters of palm oil. The world's expanding oil palm plantation areas contribute to climate change and in-return, climate is change also affecting the health of oil palms through a range of abiotic and biotic stresses. Current advancements in Precision Agriculture research using UAV gives an advantage to detect the health conditions of oil palm at early stages. Thus, remedial actions can be taken to prolong the life and increase oil palms productivity. This paper explores the use of UAV derived NDVI and CPA of young oil palm to detect the health conditions. NDVI of individual oil palm were extracted using ground masking layer from the dense point clouds and visual on-screen manual editing was done for removing trees other than oil palm in ENVI software. The classified individual crown NDVI were then processed to extract the mean NDVI also conversion to vector to obtain the individual crown outline. Extracted mean NDVI was classified into un-healthy and healthy trees while the CPA was classified into small, medium and big size classes. These classes of NDVI and CPA were analysed using GIS overlay method thus revealing the spatial patterns of individual oil palm trees and its health conditions. Overall, the majority of oil palm trees of the study area are healthy but average performing. However, few oil palm trees detected having health problems which has low NDVI and small CPA. This study demonstrates that biophysical parameters such as the CPA can be used to detect individual young oil palm trees health conditions and problems when combined with vegetation indices such as NDVI.
\end{abstract}

\section{INTRODUCTION}

Oil Palm is currently the biggest source of vegetable oil because of its high yield and long productivity with lifespan up to 25 years (Lotte et al., 2017). Malaysia, being one of the biggest producers (39\% of world) and exporters of palm oil has an important role to supply for global needs of edible oil (Malaysian Palm Oil Council).

There have been various studies that show the increasing oil palm tree health related risk due to expanding plantation area effects on climate change and also climate change effects on oil palm. Oil palm plants, like any other Tropical plants are often at the limits of growth where small changes in climate can effect its survival (Paterson \& Lima, 2018). Oil palm are affected by climate change through a range of expected abiotic (amount and repartition of rainfall, temperatures, carbon dioxide concentration, soil salinity) and abiotic (diseases, pests, pollinators, associated crops) stresses (Rival, 2017).

Currently oil palm tree health risk became an interesting topic of Precision Agriculture research because of availability of high resolution satellite data as well as UAV data. Moreover, detecting oil palm trees health at early stage is a big advantage to curb future losses from underperforming low yield trees. There could be number of reasons to low yield such as poor quality seedlings, fertilizers, climatic, edaphic factors etc. which requires further investigations.
Previous studies indicated that early detection of Oil Palm tree health conditions can assist in immediate, appropriate and effective remedial actions. This will hopefully prolong the life of Oil Palms and raise its productivity (Priwiratama, Hari \& Agus, 2014). Plant health condition can be monitored by studying the phonological changes in the plant biophysical parameters such as plant height, diameter at breast height (DBH), crown diameter, shape/size of fronds, Leaf Area Index (LAI), Crown Projection Area, NDVI etc.

In some diseases these biophysical parameters change very fast but sometimes there are no short-term changes in biophysical parameters. Advancement in remote sensing techniques with the development of UAVs based observation can reveal changes in biophysical parameters more precisely as compared to satellite based observations. In plant studies, NDVI has been used widely as an indicator of health of tree in a homogeneous vegetation species (Xiao $\&$ McPherson, 2005). Khai et al., 2017 demonstrated that increase in LAI of the tree crown show a proportional increase in the spectral reflectivity or NDVI during the initial growth stage.

Crown Projection Area (CPA) is one of the biophysical parameter that related to age (Khai et al., 2017). According to McMorrow, 2001 there is a positive linear relationship between oil palm crown projections and age for early growth stage of Oil Palm from 2-13 years old. According to Chemura et al. 2015, after 15 years of growth of oil palm 
there is no significant grown in CPA. Hence, denser crown canopy is a good indication of robust and healthy growth (Khai et al., 2017). Therefore, accurate measurement and monitoring of CPA can provide some information about the age and detect abnormalities of Oil palm trees. This study aims to explore the use of UAV derived CPA and NDVI to detect the conditions and possible health problems in young oil palm trees.

\section{STUDY AREA}

The study was conducted with permission at Ladang Sabahmas oil palm estate located in Lahad Datu, Sabah, Malaysian Borneo. The climate is Tropical with mean temperature of $27.6^{\circ} \mathrm{C}$ and average rainfall of $2,063 \mathrm{~mm}$ (en.climate-data.org). Exact location of the data collected is located between longitude of $118^{\circ} 24^{\prime} 26.299^{\prime \prime} \mathrm{E}, 118^{\circ} 24^{\prime}$ $35.717 " \mathrm{E}$ and Latitude $5^{\circ} 8^{\prime} 8.368^{\prime \prime} \mathrm{N}, 5^{\circ} 8^{\prime} 4.852^{\prime \prime} \mathrm{N}$. The study area covers 5.8 Acres and 275 young oil palm trees (more than 3 years old). According to the plantation personnel, problems such as nutrient deficiency, low quality seedlings, water stress and pest attacks are occurring within the study area. Figure 1 shows the location of the study area and UAV captured data.

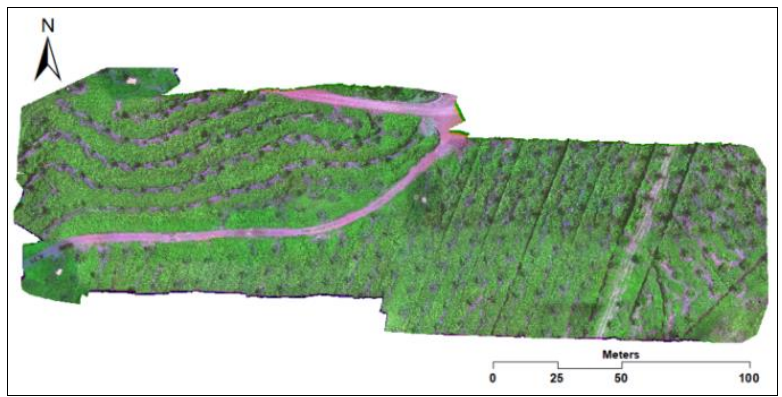

Figure 1. Study Area (MicaSense RedEdge in False Composite B, Red Edge, Red)

\section{METHODOLOGY}

In this study, the NDVI of the young Oil Palm trees and the point clouds were derived from Orthomosaic collected from a DJI Phantom 4 UAV using MicaSense RedEdge. Figure 2 shows the workflow of this study.

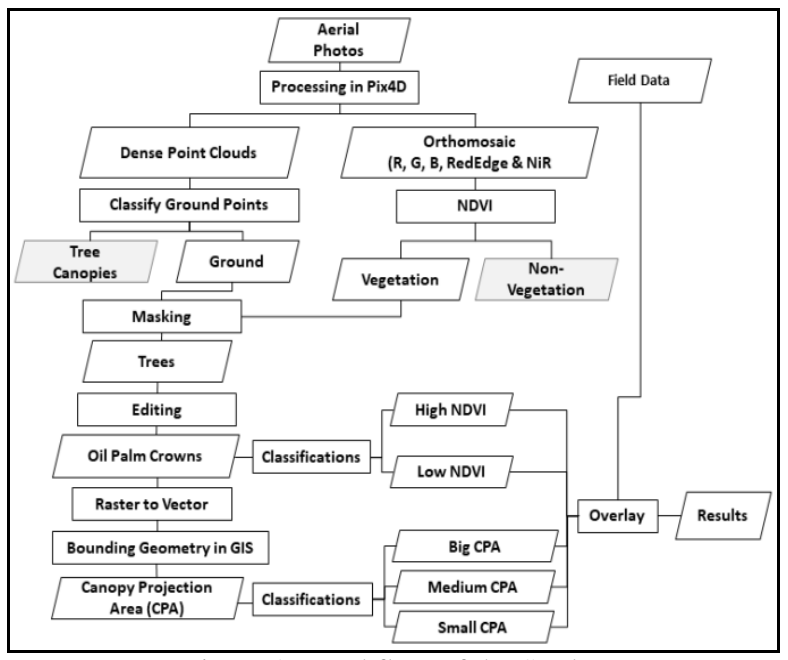

Figure 2. Workflow of the Study

\subsection{Data Collection}

The data were collected between the 29th and $30^{\text {th }}$ of August 2018. A DJI Phantom 4 series UAV mounted with MicaSense RedEdge multispectral sensor was used in the aerial photo collections. Meanwhile a GoPro Action Camera and handheld GPS were used to collect photos, location and to note the health conditions of the affected Oil Palm trees.

The MicaSense RedEdge Multispectral reflectance was calibrated on the field before flight missions. A total of four flight missions were flown and yielded a total of 8,800 high overlap aerial photos collected at $20 \mathrm{~m}$ altitude resulting $1.73 \mathrm{~cm}$ Ground Sample Distance (GSD).

\subsection{Processing}

The aerial photos processing was done using Pix4D software to produce dense point clouds and Orthomosaic Multispectral and Digital Surface Model outputs. Normalised Difference Vegetation Index (NDVI) was computed in ENVI software using the Red band Near Infrared bands of the Multispectral Orthomosaic output. The formula for NDVI as the following:

$$
\text { NDVI }=\frac{\text { NIR }- \text { red }}{\text { NIR }+ \text { red }}
$$

The dense point clouds output classification was done in Agisoft Metashape software to classify the ground and trees. Ground mask was produced for later masking of the vegetation layer from NDVI density slice. The classified ground and trees were shown as in figure 3.

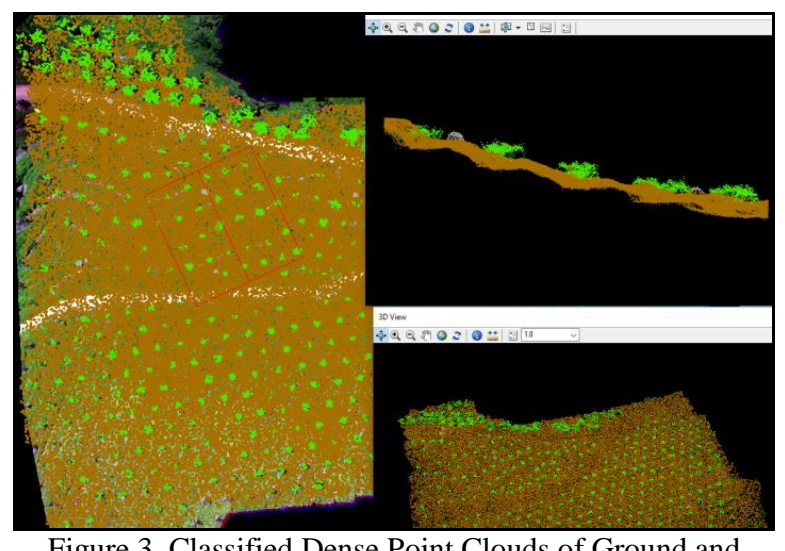

Figure 3. Classified Dense Point Clouds of Ground and Trees

Meanwhile, the computed NDVI was used to separate between vegetation and non-vegetation by using density slice threshold values ( -1 to 0 for non-vegetation and $0.1-1$ for vegetation) in ENVI.

\subsection{Oil Palm Crown Extractions}

The NDVI vegetation layer was masked using the ground layer mask produced from the dense point clouds classification to extract the individual oil palm NDVI crown layer. It was observed that the output also included other than oil palm trees. Trees other than oil palm is quite distinguishable based on its non-star crown shape and located outside of the planting row. The trees other than oil palm was visually inspected and was removed manually. 


\subsection{Raster to Vector}

The individual oil palm tree NDVI crown was converted into vector to obtain the crown vector outline for further processing and analysis using ArcMap.

\subsection{Crown Projection Area (CPA)}

The CPA was generated using the circle Minimum Bounding Geometry tools in ArcMap software and area size in meter square was computed. The extracted individual oil palm crown NDVI as shown in figure 4 below.

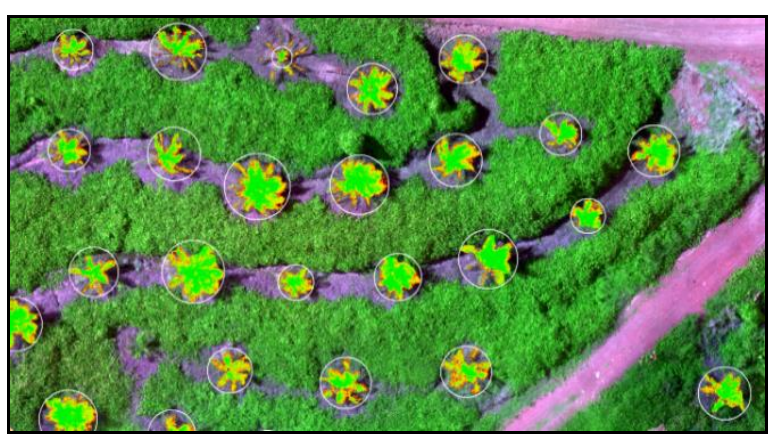

Figure 4. Individual oil palm crown NDVI

\section{RESULTS AND DISCUSSION}

Results of processing were; individual NDVI oil palm crown, individual crown vector outline and the Crown Projection Area (CPA). These parameters were used for further analysis.

\subsection{Extractions of Attributes for NDVI and CPA}

Individual Tree Crown NDVI values (min, max, range, mean and sum) were extracted using ArcMap. The CPA was generated using the individual crown outline by circle method of Minimum Bounding Geometry tools in ArcMap and the area size was also computed.

\subsection{The NDVI and CPA Classifications}

Mean NDVI was classified into two classes of un-healthy with threshold mean NDVI value below 0.40 and healthy classes above 0.40 mean NDVI. The CPA was classified into three classes of small, medium and big with almost equal range value of 10 square meters each except for big class adjusted to start at 20 to 31 square meters. This was because according to Chemura et al. 2015, the oil palm with age of 3 years and above should have developed more than 20 square meters of CPA.

\subsection{Overlay Analysis Results of NDVI and CPA}

The overlay result shows all of the 38 trees with big CPA are healthy. There are 170 medium healthy trees and only 6 medium un-healthy trees. Meanwhile there are 52 small healthy trees and 9 small unhealthy trees. The results were summarised as in table 1 .

\begin{tabular}{|c|c|c|c|c|}
\hline \multirow{2}{*}{$\begin{array}{c}\text { NDVI } \\
\text { Class } \\
\text { (Mean) }\end{array}$} & \multicolumn{4}{|c|}{ Canopy Projection Area CPA (m2) } \\
\cline { 2 - 5 } & $\begin{array}{c}\text { Small } \\
(1-10)\end{array}$ & $\begin{array}{c}\text { Medium } \\
(11-19)\end{array}$ & $\begin{array}{c}\text { Big } \\
(20 \text { to } \\
31)\end{array}$ & Total \\
\hline $\begin{array}{c}\text { Un- } \\
\text { healthy } \\
(0.3-0.40)\end{array}$ & $\begin{array}{c}9 \\
(3 \%)\end{array}$ & $\begin{array}{c}6 \\
(2 \%)\end{array}$ & $\begin{array}{c}0 \\
(0 \%)\end{array}$ & $\begin{array}{c}15 \\
(5 \%)\end{array}$ \\
\hline $\begin{array}{c}\text { Healthy } \\
(0.41-1.0)\end{array}$ & $\begin{array}{c}52 \\
(19 \%)\end{array}$ & $\begin{array}{c}170 \\
(61 \%)\end{array}$ & $\begin{array}{c}38 \\
(14 \%)\end{array}$ & $\begin{array}{c}260 \\
(95 \%)\end{array}$ \\
\hline Total & $\begin{array}{c}61 \\
(22 \%)\end{array}$ & $\begin{array}{c}176 \\
(64 \%)\end{array}$ & $\begin{array}{c}38 \\
(14 \%)\end{array}$ & $\begin{array}{c}\mathbf{2 7 5} \\
(\mathbf{1 0 0} \%)\end{array}$ \\
\hline
\end{tabular}

Table 1. Summary of the NDVI CPA Overlay Analysis

\subsection{Spatial Distributions of NDVI CPA Overlay Analysis Results}

It was revealed that trees with big CPA and healthy NDVI are clustered at the flat land in the east side of the study area. Meanwhile the medium CPA healthy and un-healthy trees are scattered from middle to west part of the study area. The small CPA of healthy and un-healthy trees are scattered in between the medium CPA trees. The spatial distributions of the trees are shown in figure 5 below.

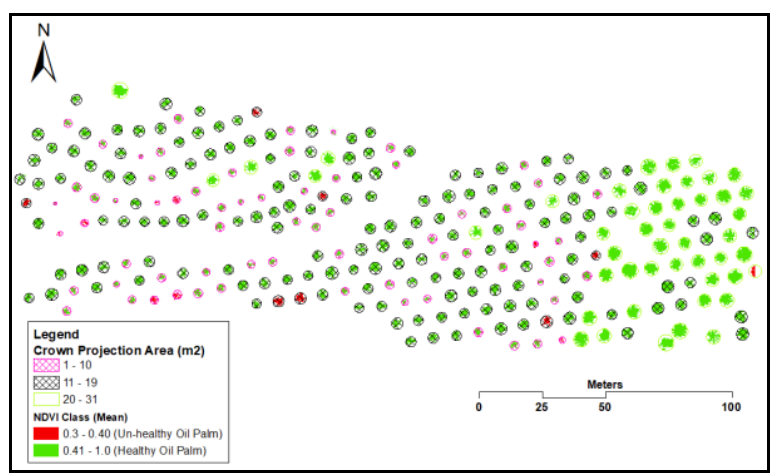

Figure 5. Spatial Distributions of Oil Palm Health

\subsection{Interpretations on the NDVI CPA Analysis Results}

Based on the literature and findings indicated by the results, it can be understood that combinations of NDVI CPA attributes can be interpreted as following scenarios of oil palm health conditions;

1. Oil palm trees with big CPA and having healthy NDVI are well performing trees.

2. Oil palm trees with medium CPA but healthy NDVI are average performing.

3. Oil palm trees with small CPA and healthy are most probably trees having stress resulting stunted growth.

4. Lastly oil palm with medium to small CPA and un-healthy NDVI most probably are trees having disease or pest problem.

Overall, it was observed that the study area has more than half or $61 \%$ of the total oil palm trees are average performing and the majority are healthy $(95 \%)$.

\subsection{Limitations and Future Directions of the Study}

There are two limitations identified within this study. Firstly, the limitation of crown extractions method whereas the output included trees other than oil palm. Output quality control and validation still subjected to manual visual inspections on the high resolution orthomosaic also using 
field data and manual editing are required to clean the extracted crown layer. The second limitation is the confirmation on the specific individual tree health conditions which requires further investigation and study. For future study the authors are planning to look into the details of the health conditions also to study the feasibility of this method to be applied for wider area of the plantations.

\section{CONCLUSION}

This study shows biophysical parameters such as the CPA can be used to detect individual young oil palm trees health conditions and problems when combined with vegetation indices such as NDVI. There are 15 unhealthy trees out of 275 in the study area. This information can be useful to closely monitor the factors that influence the plant health. In addition to health conditions, the study output is also useful for other purposes of oil palm studies such as tree counting and inventory.

\section{ACKNOWLEDGEMENTS}

This study is part of the on-going Msc. research by Mr. Mohd Shahrizan Bin Syukur on oil palm health using applications of remote sensing and geospatial. The authors would like to thanks the Management and staffs especially the managers; Mr. Asrif Mahmud and Mr. Henry Jubair of Ladang Sabahmas, Lahad Datu, Sabah Malaysian Borneo for the cooperation which enabled this research to be done.

\section{REFERENCES}

en.climate-data.org, climate of Lahad Datu. https://en.climate-data.org/asia/malaysia/sabah/lahad-datu54128/ Accessed on 31 May 2019.

Khai Loong Chong, Kasturi Devi Kanniah, Christine Pohl \& Kian Pang Tan, (2017). A review of remote sensing applications for oil palm studies. Ge0-spatial Information Science, Vol. 20, No. 2, 184-200.

Kian Pang Tan, Kasturi Devi Kanniah \& Arthur Philip Cracknell, (2014). On the upstream inputs into the MODIS primary productivity products using biometric data from oil palm plantations. International Journal of Remote Sensing, $35: 6,2215-2246$

Lotte S. Woittiez, Mark T. van Wijk, Maja Slingerland, Meine van Noordwijk \& Ken E. Giller, (2017). Yield gaps in oil palm: A quantitative review of contributing factors. Europ. J. Agronomy, 83: 57-77.

Malaysian Palm Oil Council (MPOC), Malaysian Palm Oil Industry

http://www.mpoc.org.my/Malaysian_Palm_Oil_Industry.asp x Accessed on 31 May 2019.

McMorrow, J., (2001). Linear Regression Modelling for the Estimation of Oil Palm Age. International Journal of Remote Sensing. 22 (12), 2243-2264.

Paterson R. R. M. \& Lima N., (2018). Climate Change Affecting Oil Palm Agronomy, and Oil Palm Cultivation
Increasing Climate Change, Require Amelioration. Ecology and Evolution. 8:452-461.

Priwiratama, Hari, and Agus Susanto, (2014). Utilization of Fungi for the Biological Control of Insect Pests and Ganoderma Disease in the Indonesian Oil Palm Industry. Journal of Agricultural Science and Technology 4: 103-111.

Rival, A. (2017). Breeding the oil palm (Elaeis guineensis Jacq.) for climate change. Oilseeds \& Fats Crops and Lipids, 24

Xiao, Qingfu \& Mcpherson, E. (2005). Tree health mapping with multispectral remote sensing data at UC Davis, California. Urban Ecosystems. 8. 349-361.

Revised August 2019 